\title{
Improving Clock Accuracy, Bronze Age Style
}

\author{
Researchers may have found the earliest example of humans tinkering \\ with the workings of a clock to improve its timekeeping accuracy.
}

\section{By Katherine Wright}

$\square$ eveloping precise and stable devices for timekeeping is an integral part of physics research. Accurate ticks are essential for everything from improving satellite navigation to understanding the daily life cycles of plants. But the clocks that we use today are still relatively new inventions-the pendulum clock, a physics favorite, turned just 365 years old this year, a tiny fraction of the 300,000 years during which modern humans have roamed the planet. Before resonating atoms, winding gears, and swinging weights, humans used water and then the Sun to measure the passing of time. Now, a trio of archaeologists and physicists say that they have identified the first known example of an ancient clock that uses both elements. The researchers hypothesize that our

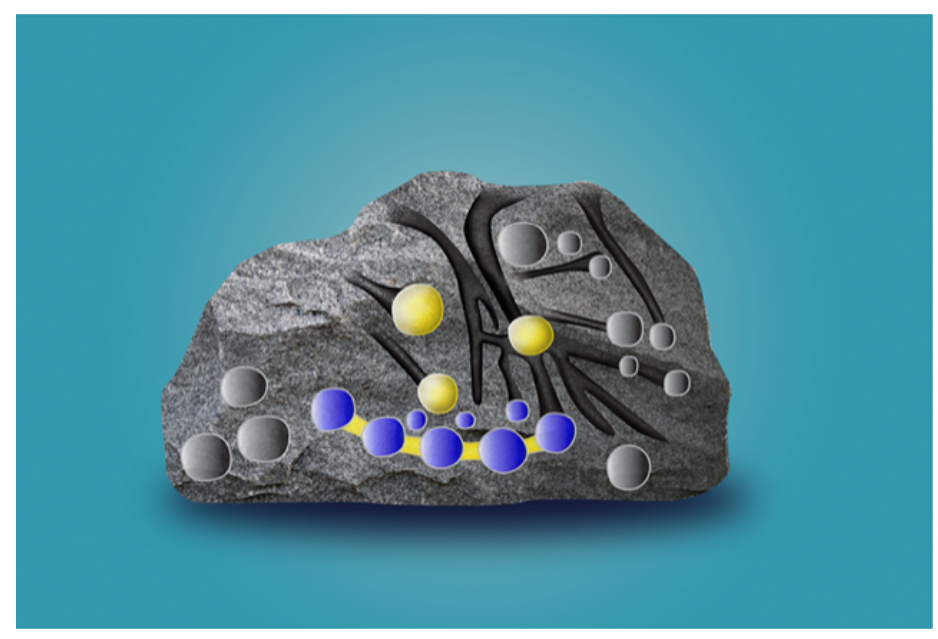

Cartoon of the rock clock. The blue pits were likely used to tell the time with water, while the yellow grooves made up the sundial. Together they make up the "smiling" pattern referenced in the text. Credit: APS/Carin Cain prehistoric colleagues landed on the combined design as part of an early effort to improve timekeeping accuracy.

The clock at the center of the claim comprises a series of carvings in a slab of limestone rock. Round pits and elongated grooves pockmark its weathered surface, with several different patterns visible. On one area of the rock, long grooves connect small pits, creating a flower pattern. In another area, an arrangement of pits and short grooves appears to trace the outline of a smiling face. Those patterns do not match the etchings of other known ancient sundials or water clocks.

While plowing a field at a vineyard in Pyatikhatki, Russia, in 1982, a farmer uncovered the rock clock. Archaeologists have dated the relic to $2500-1500 \mathrm{BCE}$. Other local artifacts that date to the same time period include stone tombs that have holes, grooves, and other patterns carved into their sides in a similar style-but without a known functionality.

Archaeologists have unearthed other rock clocks in this region. But those examples contain only small round pits and no grooves between the pits as in the Pyatikhatki specimen, says Larisa Vodolazhskaya, an astrophysicist turned archaeologist who studies both topics at the Southern Federal University in Russia. Vodolazhskaya learned of the rock clock in 2019 and teamed up with Andrey Novichikhin, an archaeologist at Anapa Archaeological Museum, also in Russia, to investigate how the rock clock might have worked.

At first glance, Vodolazhskaya says, the "mouth" of the clock's face-like carving pattern reminded her of a so-called analemmatic sundial that still exists today-one where the hour markers are arranged in an ellipse. The pits forming the eyes 


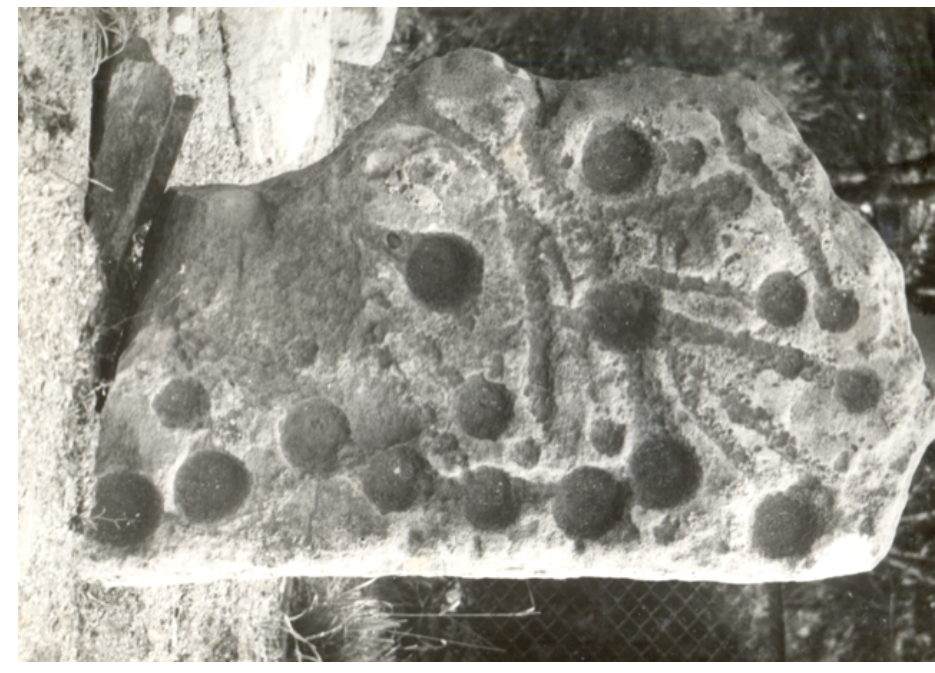

Researchers think that a rock found in Russia has both a water clock and a sundial carved into it, the only known example of such an artifact.

Credit: Courtesy of L. Vodolazhskaya

and nose, she thought, might have been used to mount the gnomon (the structure that casts the shadow). But, carrying out calculations, she soon realized that there was more to this clock than she had initially thought.

Vodolazhskaya's calculations indicate that the hour markers are not defined by the pits that line the smile's curve but rather by the grooves that link the pits together. (The positions for the nose and eyes do seem to fit with the expected positions for the mount markers for the gnomon.) The smile's pits, instead, have the appropriate dimensions to function as the hour markers of a different kind of clock known to have existed since at least the 16th century BCE-one that uses water to tell the time. In these clocks, the passing of time is measured by the drips of water into a container. The water's level relative to markings on the container indicate the passing of hours. The team's measurements show that each pit holds, on average, $160 \mathrm{ml}$ of water, close to the amount commonly used in water clocks of this period. The three tiny holes just above the smile likely held pebbles that were used to count hours for water clocks.

Archaeologists generally believe that the water clock was invented before the sundial. As such, Vodolazhskaya and her colleagues hypothesize that their relic shows this Bronze Age community transitioning between the two technologies. Both sets of markings seem to have been carved into the rock at the same time, she says, with both clocks likely being used simultaneously to tell the time. The presence of both clocks "made it possible to compare the accuracy of the two methods to tell the time," she says.

Using the motion of the Sun rather than the dropping of water is a more precise way to tell the time, Vodolazhskaya says. The Sun's movement across the sky is relatively stable over time, but it is hard to carve two pits of identical volume into a sheet of rock, for example. The caveat, Vodolazhskaya notes, is that while sundials are more accurate than water clocks, they only work on clear days between sunrise and sunset. Having both clocks on the same rock meant that on cloudy days or at night the slab could still be used to tell the time, she says. Thus, the physics-archaeology team believes that the smiling rock clock is an important artifact in understanding the history of the development of clocks by humans.

Katherine Wright is the Deputy Editor of Physics. 